\title{
Finite Element Simulation of Chimney Emissions: A Proposal for Near Field Impact Assessment in Highly Complex Terrains
}

\author{
A. Pérez-Foguet $\uparrow$, A. Oliver $\uparrow$, J.M. Escobar + and E. Rodríguez $\ddagger$ \\ $\uparrow$ LaCàN, Department of Applied Mathematics III \\ ETS Ingenieros de Caminos, Canales y Puertos, UPC, Barcelona, Spain \\ + University Institute of Intelligent Systems and Numerical Applications in \\ Engineering (IUSIANI), University of Las Palmas de Gran Canaria, Spain
}

\begin{abstract}
This work focuses in the simulation of transport and reaction of pollutant emissions from high stacks with the Finite Element Method. Nowadays tendencies on numerical modelling of punctual emission sources are analyzed. The so-called hybrid models are one of the best available options. These models simulate the pollutant evolution in the microscale, from hundreds of meters to few kilometres, with lagrangian puff models, and the urban and regional scales, few kilometres and above, with Eulerian finite differences schemes. Approximation exposed in this paper has the aim of substituting the lagrangian local treatment, source of several problems and approximating errors, with an Eulerian Finite Element approach. Simulations for highly demanding problems (from both topography and wind speed point of view) have been computed and analyzed. The results show that the approach is efficient and accurate, although some mesh adjustments has found needed for extremely windy situations.
\end{abstract}

Keywords: atmospheric pollution, puff models, dimensionless analysis, 3D Eulerian analysis, finite element method, linear and nonlinear chemistry.

\section{Introduction}

Numerical simulation of pollutant transport and reaction on atmosphere has been a field of important advances in last thirty years, and it is still a scientific area of high interest. Acid rain, ozone, particle matter and toxic emissions are some of the key areas of analysis. Simulations are done with the so-called Air Quality Modelling Systems, which three principal components are the emission model, the meteorological model and the transport and photochemical models. First one is used to characterize different source emissions of chemical compounds of interest, second one is used to 
determine atmospheric physical characteristics as wind and temperature fields, and the third one is used to simulate how pollutants transport by advection and diffusion, how react among them, and how, eventually, are deposited on the terrain.

Environmental Protection Agency of United States, www.epa.gov, classifies Air Quality Modelling Systems on three groups: Dispersion, Photochemical, and Reception. Dispersion models are used to estimate pollution levels at ground level and near punctual sources. Photochemical are used to estimate the impact of all the sources in an specified zone of different concentrations, and the deposition along long distances. Reception models are used to identify and characterize possible source based on receptors and sensors measures.

Main dispersion models are plume models [1], particle tracking models [2] and puff models $[3,4,5]$. All three are based on a lagrangian approach and usually includes first order or linearized chemical and photochemical models. Versions with nonlinear reaction descriptions can be found.

In contrast with dispersion models, photochemical ones follow an eulerian description of the problem, discretized, typically, with finite differences schemes [6]. Spatial domains are about few hundreds of kilometres by side with distretizations of 16 or 32 kilometers. Areas of interest (usually of few tens of kilometres by side) are simulated with more detail using nested sub-grids. The horizontal resolution of nested sub-grids is two or four kilometers, although even one kilometer application can be found $[7,8,9]$. Vertical range vary from 4 to 10 kilometres with non-uniform discretitzations of 10 to 20 levels, including also, in some cases, nested sub-grids. Therefore, problems involve easily tens of thousands of grid points, and up to 100 thousand grid points are usual. Most advanced photochemical models include punctual emission treatment with puff models embedded within. These models are known as hybrid models. Examples of them are CAMx [10], UAM-V [11] and CMAQ [12].

The application of dispersion and photochemical models in environmental management has had very different focus. Former ones have been usually applied to punctual emission impact assessments, and second ones to regional planning and monitoring; thus, with a clearly different application scale and photochemical model complexity. However, some references about the need of coupling punctual emissions with regional planning with hybrid photochemical models can be found $[7,8,13]$. They are founded in the importance of coupling among key chemical components as ozone, nitrogen, and Volatile Organic Components, VOC, [14, 15], and the increasing awareness about the socioeconomic impacts of punctual emissions and pollution [16]. Although hybrid models can couple scales about one kilometre with regional ones, several limitations to this approach has been reported [7]. Thus, the search of alternatives is justified.

In this work, the use of the Finite Element Method with unstructured and adapted meshes is proposed for simulations at neighborhood and urban scales [13], up to tens of kilometers. The aim of this proposal is to present an alternative to embedded lagrangian approaches of hybrid models. A zone of 15.6 by 18 kilometers in plant and 9 in height of La Palma island (Spain) is considered as a reference problem. It involves 
a highly complex topography, from sea level to up 2300 meters, see figure 1 . A cylindrical stack of 200 meters height, 60 meters diameter on ground level and 40 on top, is included as an example of punctual emission source.

Three remarkable applications of non-structured meshes to atmospheric pollution problems can be found: 2D global-regional examples [17, 18], 3D regional examples with element refinement down to two kilometers [19], and 3D local wind simulations with element sizes ranging from two meters up to two kilometers [20, 21, 22]. Last approach is the one used in this work, although here it is also applied to simulate transport - reaction problems. Two wind fields are taken as reference: case I and case II. They correspond to situations of strong and extremely strong wind, respectively. None of both incorporate yet plume over-elevation due to emission, see [21] for recent advances on that topic.

The transport - reaction problems are solved using specific splitting schemes and numerical solvers for time integration due to the high nonlinearity of photochemical reaction models. In this work, three simplified reaction models are presented, one of them linear. The Least Squares approach with Crank-Nicolson time integration is proposed for the linear model $[23,24,25,26]$. The system of equations is solved with a preconditioned conjugate gradient scheme with incomplete Cholesky factorization [27]. First-order and Strang splitting between transport and reaction are proposed for nonlinear models [28]. The approach for linear models is used for transport steps and uncoupled node by node second order Rosenbrock time integration scheme is used for the reaction ones [29].

In the following, after description of main characteristics of the models considered, solution of the reference problem is presented. First, a detailed analysis of spatial discretization and the two wind fields considered is summarized. After that, numerical solutions of the two transport and reaction problems are presented. The influence of time step size in solution quality is specifically analyzed. The work finishes with most relevant conclusions.

\section{Model characteristics}

The modelling approach followed in this work separate wind and transport - reaction simulations. First, using a wind model, a wind field is approximated in full domain from some puntual data. A mass consistent wind model with a log-linear wind profile is used. A mesh adapted to terrain curvature is used for wind field computation. The wind field and the mesh used are input data for transport - reaction problem, which is solved next.

In the following, after a brief review of wind model and discretization algorithm, three photochemical reaction models are analyzed. First a linear first order chemical reaction model, which leads to a linear problem, and after that, two of the most simple nonlinear models referenced in the literature. Presentation of linear model focuses in the dimensionless analysis of the problem and of the nonlinear one in the specific 


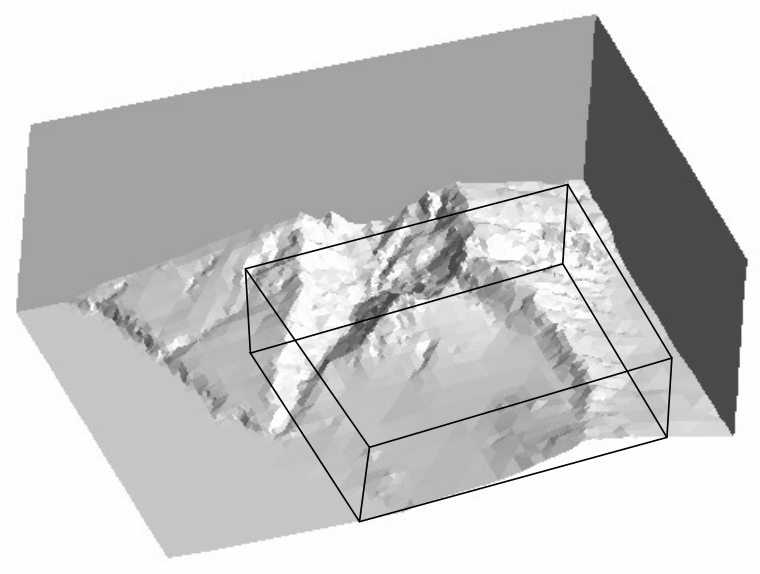

Figure 1: Sketch of full domain and emission and transport zone, defined by coordinates $209000<x<225500, y<3179000$ and $z<3000$.

numerical requirements due to nonlinearity.

\subsection{Wind model and spatial discretization}

A mass consistent model for wind field adjustment is proposed. It is based on the continuity equation, the assumption that the air density is constant and the impermeability conditions on the terrain $\Gamma_{R}$. Following equations define the model

$$
\begin{array}{ll}
\nabla \cdot \mathbf{u}=0 & \text { in } \Omega \\
\mathbf{n} \cdot \mathbf{u}=0 & \text { on } \Gamma_{R}
\end{array}
$$

where $\nabla$ is the gradient with respect to the spatial coordinates $\mathbf{x}, \mathbf{u}$ is the wind velocity vector, $\mathbf{n}$ the unitary normal vector to the boundary and "." the standard scalar product. A least-square problem is proposed to adjust $\mathbf{u}$ to the observed wind $\mathbf{u}_{\mathbf{0}}$, subject to equations (1-2). Lagrange multiplier technique is used to solve this problem, whose minimum comes to form the Euler-Lagrange equations and yields an elliptic equation and boundary conditions in the Lagrange multiplier $\phi$

$$
\begin{aligned}
\nabla \cdot(\mathbf{T} \nabla \phi) & =-\nabla \cdot \mathbf{u}_{\mathbf{0}} & & \text { in } \Omega \\
\phi & =0 & & \text { on } \partial \Omega / \Gamma_{R} \\
\mathbf{n} \cdot \mathbf{T} \nabla \phi & =-\mathbf{n} \cdot \mathbf{u}_{0} & & \text { on } \Gamma_{R}
\end{aligned}
$$

where $\mathbf{T}$ is the diagonal transmissivity tensor. 
A discretization with tetrahedral finite elements, obtained with the mesh generator described in [30], is used for solving the above problem. Meshes obtained using this technique can be adapted to the topography of a rectangular area with minimal user intervention. The mesh generation starts with an efficient and adaptive point generation. Points are distributed in the domain under study in such a way that preserves the topographic information of the terrain with a decreasing density as altitude increases. Refinement/derefinement techniques in 2-D and vertical spacing function are used in point distribution. Next, with the aid of an auxiliary parallelepiped, a proceeding based on Delaunay triangulation has been set forth to generate the mesh automatically, assuring conformity with the terrain surface. Transformation from auxiliary parallelepided to real topography can produce poor quality elements, or even tangled ones. For this reason a procedure to optimize the mesh is applied [31]. This technique increases the mesh quality and untangle it, at the same time.

In order to obtain the observed wind, horizontal interpolation of the station measures is carried out. Then, a log-linear wind profile is built up to the surface layer taking into account the horizontal interpolation, the effect of roughness on the wind velocity and air stability. Above the surface layer, a linear interpolation is carried out using the geostrophic wind. For more details see [20]. Both horizontal and vertical interpolations involve some parameters that typically take their values using an heuristic approach. Nowadays, genetic algorithms can be used in order to obtain those values automatically [22].

\subsection{Transport - reaction model}

A convection - diffusion - reaction system of equations defines the transport - reaction model:

$$
\frac{\partial \mathbf{c}}{\partial t}+\mathbf{u} \cdot \nabla \mathbf{c}=\nabla \cdot(\mathbf{K} \nabla \mathbf{c})+\mathbf{e}+\mathbf{s}(\mathbf{c})
$$

with $(\mathbf{x}, t) \in \Omega \times\left(0, t^{e n d}\right]$, the initial condition $\mathbf{c}(\mathbf{x}, 0)=\mathbf{c}^{i n i}(\mathbf{x})$ on $\mathbf{x} \in \Omega$, and the following boundary conditions:

$$
\begin{cases}\mathbf{c}(\mathbf{x}, t)=\mathbf{c}^{e m i}(\mathbf{x}) & \text { in } \Gamma_{D}: \text { Stack source point } \\ \mathbf{n} \cdot \mathbf{K} \nabla \mathbf{c}=-\mathbf{V}^{d} \mathbf{c} & \text { in } \Gamma_{R}: \text { Terrain } \\ \mathbf{n} \cdot \nabla \mathbf{c}=0 & \text { in } \Gamma_{N}=\partial \Omega \backslash\left(\Gamma_{D} \cup \Gamma_{R}\right)\end{cases}
$$

where $t$ is time; $\mathbf{c}$, e and $\mathbf{s}(\mathbf{c})$ the concentration, emission and source vectors, respectively, all of them with a dimension $n_{c} ; \mathbf{K}$ the diffusion matrix, of dimension $3 n_{c}$; and $\mathbf{V}^{d}$ the dry deposition matrix, of dimension $n_{c}$ and diagonal with terms $\left[\mathbf{V}^{d}\right]_{i i}=V_{i}^{d}$, $i=1, . ., n_{c}$. In equations (1-2), the scalar product "." is applied $n_{c}$ times: the first argument multiplied by each $n_{c}$ part of the second argument.

Source term, s(c), includes chemical and photochemical reactions and wet deposition processes. The complete description of photochemical reaction of atmospheric species it is highly complex. A detailed VOC decomposition involves hundreds of thousand reactions, which have to be reduced using special methodologies. Actual 
reference models for gaseous phase reactions involve some tens of especies One of the most simplified involves just ten reactive species [6, 24]. Additionally, depending on the application, aqueous phase reactions can be also necessary, which involve several other reactions and species. The most simplified model used to simulate both processes, aqueous and gaseous, involve nonlinear reaction of four species [5]. A linearized implementation of it is used in puff models embedded into hybrid models. Finally, remark that some situations are modelled using just first order chemistry, which leads to linear models.

In the following, main characteristics of linear and nonlinear reaction models are presented. First part, linear reaction, focuses in dimensionless analysis of the problem. Second one, nonlinear reaction, focuses in the numerical characteristics of two specific source terms expressions from the point of view of both computation itself and of their derivatives.

\subsubsection{Linear reaction}

Linear reaction is characterized by $\mathbf{s}(\mathbf{c})=\mathbf{A} \mathbf{c}$, with $\mathbf{A}$ a matrix of dimension $n_{c}$. A simple model is considered for dimensionless analysis of the problem: A two species model, $n_{c}=2$, with reaction matrix verifying $[\mathbf{A}]_{11}=-[\mathbf{A}]_{12}=-A$ and $[\mathbf{A}]_{12}=$ $[\mathbf{A}]_{22}=0$, thus, neglecting wet deposition. Diffusion matrix is considered diagonal on work coordinates and equal on both species, with values $K_{h}$ on horizontal and $K_{z}$ on vertical axes. Additionally, null values are considered for initial conditions, $\mathbf{c}^{i n i}=0$, emission of secondary specie through boundary, $\mathbf{c}_{2}^{e m i}=0$, and emissions inside the domain, $\mathbf{e}=0$.

Equations (6-7) can be expressed in dimensionless form using as a reference values concentration vector $\mathbf{C}=\left(\left[\mathbf{c}^{e m i}\right]_{1},\left[\mathbf{c}^{e m i}\right]_{1}\right)$, velocity $V$, time $T$ and length $L=V T$. Under the hypothesis of former paragraph the resultant dimensionless problem is:

$$
\frac{\partial \widetilde{\mathbf{c}}}{\partial \widetilde{t}}+\widetilde{\mathbf{u}} \cdot \widetilde{\nabla} \widetilde{\mathbf{c}}=\widetilde{\nabla} \cdot(\widetilde{\mathbf{K}} \widetilde{\nabla} \widetilde{\mathbf{c}})+\widetilde{\mathbf{A}} \widetilde{\mathbf{c}}
$$

for $(\widetilde{\mathbf{x}}, \widetilde{t}) \in \widetilde{\Omega} \times(0, \widetilde{t} f i n]$, with initial condition $\widetilde{\mathbf{c}}(\widetilde{\mathbf{x}}, 0)=0$ for all $\widetilde{\mathbf{x}} \in \widetilde{\Omega}$, and boundary conditions:

$$
\begin{cases}\widetilde{\mathbf{c}}(\widetilde{\mathbf{x}}, t)=(1,0) & \text { in } \widetilde{\Gamma}_{D}: \text { Stack source point } \\ \mathbf{n} \cdot \widetilde{\mathbf{K}} \widetilde{\nabla} \widetilde{\mathbf{c}}=-\widetilde{\mathbf{V}}^{d} \widetilde{\mathbf{c}} & \text { in } \widetilde{\Gamma}_{R}: \text { Terrain } \\ \mathbf{n} \cdot \widetilde{\nabla} \widetilde{\mathbf{c}}=0 & \text { in } \widetilde{\Gamma}_{N}=\partial \Omega \backslash\left(\Gamma_{D} \cup \Gamma_{R}\right)\end{cases}
$$

where $\widetilde{\mathbf{x}}=\mathbf{x} / L, \widetilde{t}=t / T, \widetilde{\nabla}$ is the gradient respect $\widetilde{\mathbf{x}}, \widetilde{\mathbf{c}}_{i}=\mathbf{c}_{i} / \mathbf{C}_{i}$ for $i=1,2$, $\widetilde{\mathbf{u}}=\mathbf{u} / V, \widetilde{\mathbf{K}}=T \mathbf{K} / V^{2}, \widetilde{\mathbf{A}}=T \mathbf{A}$ and $\widetilde{\mathbf{V}}^{d}=\mathbf{V}^{d} / V$. Dimensionless parameters are $\widetilde{K}_{h}=T K_{h} / V^{2}, \widetilde{K}_{v}=T K_{v} / V^{2}, \widetilde{A}=T A, \widetilde{V}_{1}^{d}=V_{1}^{d} / V$ y $\widetilde{V}_{2}^{d}=V_{2}^{d} / V$.

Given $V$ and $T$, a simple physical interpretation of problem can be done. Let us consider a reference problem with an uniform velocity field of magnitude $V$, fix $T=A^{-1}$ and do not consider neither diffusion nor dry deposition effects. In this situation, $L$ can be interpreted as the distance necessary to reduce the concentration of an 
emitted mass differential to the $37 \%$ of the emitted concentration (exactly $100 e^{-1} \%$, solution of the corresponding Lagrangian linear ordinary differential equation on the streamlines with origin in the emission source).

The material parameters of the problem considered in this work are $K_{h}=100$ $\mathrm{m}^{2} / \mathrm{s}, K_{v}=50 \mathrm{~m}^{2} / \mathrm{s}, \alpha=0.0012 \mathrm{~s}^{-1}, V_{1}^{d}=0.013 \mathrm{~m} / \mathrm{s}$ and $V_{2}^{d}=0.054 \mathrm{~m} / \mathrm{s}$. A value of $T=800 \mathrm{~s}$ implies $\widetilde{A}=1$, and with $V=40 \mathrm{~m} / \mathrm{s}$, it is found that $L=32000 \mathrm{~m}$, $\widetilde{K}_{h}=8 \cdot 10^{-5}, \widetilde{K}_{v}=4 \cdot 10^{-5}, \widetilde{V}_{1}^{d}=3 \cdot 10^{-4}$ y $\widetilde{V}_{2}^{d}=1 \cdot 10^{-3}$. With half reference velocity, $V=20 \mathrm{~m} / \mathrm{s}, L$ is divided by two, thus it is equal to $16000 \mathrm{~m}, \widetilde{K}_{*}$ is multiplied by four, thus of order $10^{-4}$, and $\widetilde{V}_{*}^{d}$ by two, thus of order $10^{-3}$. Under these assumptions, the problem is basically convective, dry deposition is negligible, and, following former interpretation, 16 or 32 kilometers are needed to have $37 \%$ of inicial concentration as result of reaction following streamlines, depending on reference velocity. Further analysis of influence of material parameters in the results can be done with dimensionless formulation of the model.

\subsubsection{Nonlinear reaction}

Nonlinear reaction models are characterized by nonlinear vectorial function $\mathbf{s}(\mathbf{c})$. As commented in the introduction, to deal with nonlinearity the partial differential operator is splitted in into two: One including convection and diffusion terms and other the reaction. Time integration of reaction term is done uncoupled node by node, thus the solution of a large system of equation is not necessary. However, a linear system of dimension $n_{c}$ involving the Jacobian matrix $\frac{\partial \mathbf{s}}{\partial \mathbf{c}}$ has to be solved in each time step and for all mesh nodes, thus specific attention is devised. The characteristics of two of the simplest nonlinear reaction models are summarized in the following.

First, the RIVAD model [5], which is defined as a pseudo-first-order chemical scheme for acid rain simulation. It is specially calibrated for non-urban areas, where the concentration of VOC is reduced. It involves four species, and the source term is given by: $\mathbf{s}_{1}(\mathbf{c})=-\mathbf{s}_{2}(\mathbf{c})=-\alpha_{1}(\mathbf{c}) \mathbf{c}_{1}$, and $\mathbf{s}_{3}(\mathbf{c})=-\mathbf{s}_{4}(\mathbf{c})=-\alpha_{3}(\mathbf{c}) \mathbf{c}_{3}$, with $\alpha_{1,3}(\mathbf{c})=\gamma_{1,3} /\left(\mathbf{c}_{1}+\delta_{1,3} \mathbf{c}_{3}\right)$. Note that in zones where $\mathbf{c}_{1}$ and $\mathbf{c}_{3}$ are zero or close to, both $\alpha_{1,3}(\mathbf{c})$ and $\frac{\partial \alpha_{1,3}}{\partial \mathbf{c}} \propto\left(\mathbf{c}_{1}+\delta_{1,3} \mathbf{c}_{3}\right)^{-2}$ requiere a proper numerical treatment in order to avoid excessively high reaction rates. Figure 2 shows the function $1(x+B)$ for different values of $B$. Depending on $B$ the value and slope of the function near zero varies significantly. Low values of $B$ implies extremely high values of the function itself and its derivative. The limit $\alpha_{1,3}(\mathbf{c}) \leq \alpha_{R \max }$ is imposed.

And second, the simplified photochemical scheme presented in [6], which is a ten species model with a source term quadratic in $\mathbf{c}$. The reaction term can be expressed as $\mathbf{s}_{k}(\mathbf{c})=\sum_{i=1}^{n_{c}}\left(\sum_{j=1}^{n_{c}} \alpha_{i j}^{k} \mathbf{c}_{j}+\beta_{i}^{k}\right) \mathbf{c}_{i}$, for $k=1, \ldots, n_{c}$, and with $\alpha_{i j}^{k}$ and $\beta_{i}^{k}$ model constants; And the Jacobian matrix is linear and given by $\frac{\partial \mathbf{s}_{k}}{\partial \mathbf{c}_{l}}=\sum_{i=1}^{n_{c}}\left(\alpha_{i l}^{k}+\alpha_{l i}^{k}\right) \mathbf{c}_{i}+$ $\beta_{l}^{k}$. Thus, in contrast with RIVAD model, this second one does not represent any problem for $\mathrm{c}$ equal zero or close to. 


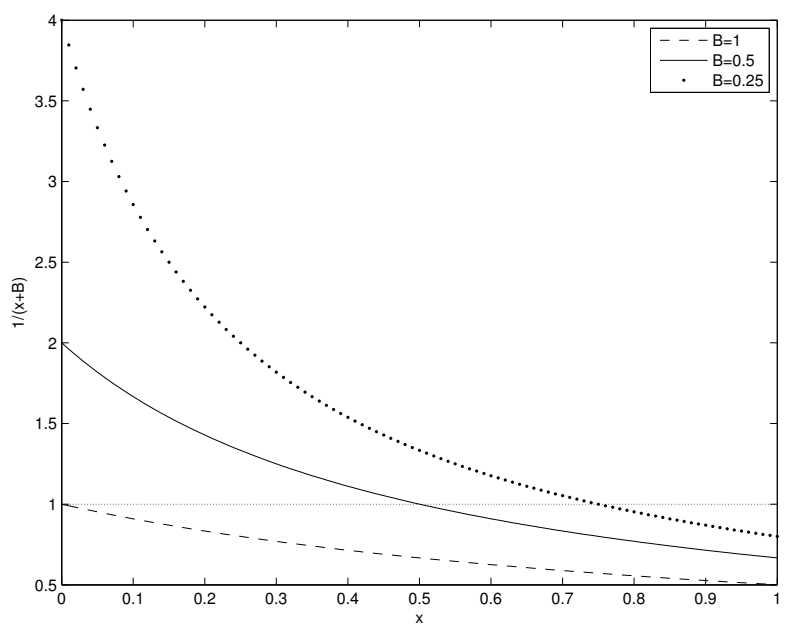

Figure 2: Relationship $1 /(x+B)$ for $x \in[0,1]$ and different values of $B$.
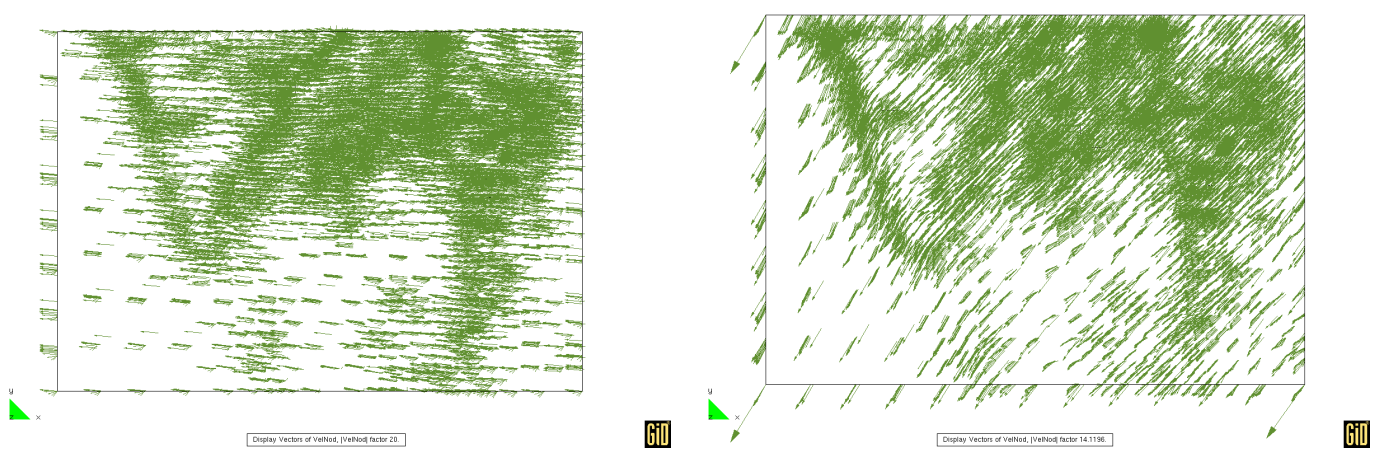

Figure 3: Top-down view of the two wind fields, case I (left) and case II (right), defined in mesh nodes.

\section{Mesh and wind analysis}

The main characteristics of mesh and wind fields used as examples in this work are shown in this section. Analysis presented here orientate numerical simulations shown in the following section. Specifically, some references for the time step are established based on mesh and wind field analysis.

Simulations of this work have been done with one spatial discretization and two wind fields computed from experimental data, cases I and II, following the model scheme presented in previous section. A non-structured mesh of 153085 thetahedral elements and 28387 nodes is used. Remarkably it includes a detailed description of emission stack geometry (two meters of element size on top of the stack). Figure 3 presents a top-down view of the two wind fields considered.

Table 1 summarizes maximum, minimum, mean, and median values of 4 interest 


\begin{tabular}{|c|c|c|c|c|}
\hline & Max. & Min. & Mean & Median \\
\hline$\Delta x(\mathrm{~m})$ & 2692. & 2.3 & 385. & 309. \\
\hline$\left\|\mathbf{u}^{I}\right\|(\mathrm{m} / \mathrm{s})$ & 45. & 0.48 & 26.5 & 26.2 \\
$\left\|\mathbf{u}^{I I}\right\|(\mathrm{m} / \mathrm{s})$ & 119. & 0.96 & 41.8 & 44.4 \\
\hline$\left\|\mathbf{u}^{I}\right\| / \Delta x\left(\mathrm{~s}^{-1}\right)$ & 11.0 & 0.0039 & 0.14 & 0.078 \\
$\left\|\mathbf{u}^{I I}\right\| / \Delta x\left(\mathrm{~s}^{-1}\right)$ & 25.8 & 0.0033 & 0.264 & 0.133 \\
\hline$\left\|\mathbf{u}^{I}\right\| \Delta x\left(\mathrm{~m}^{2} / \mathrm{s}\right)$ & 105022. & 4.2 & 11138. & 8061. \\
$\left\|\mathbf{u}^{I I}\right\| \Delta x\left(\mathrm{~m}^{2} / \mathrm{s}\right)$ & 167741. & 11.8 & 16941. & 13427. \\
\hline
\end{tabular}

\begin{tabular}{|c|c|c|c|c|}
\hline & Max. & Min. & Mean & Median \\
\hline$\Delta x(\mathrm{~m})$ & 2296. & 2.3 & 348. & 323. \\
\hline$\left\|\mathbf{u}^{I}\right\|(\mathrm{m} / \mathrm{s})$ & 43.2 & 0.48 & 23.9 & 21.9 \\
$\left\|\mathbf{u}^{I I}\right\|(\mathrm{m} / \mathrm{s})$ & 94.8 & 1.70 & 39.8 & 42.4 \\
\hline$\left\|\mathbf{u}^{I}\right\| / \Delta x\left(\mathrm{~s}^{-1}\right)$ & 11.0 & 0.0049 & 0.28 & 0.076 \\
$\left\|\mathbf{u}^{I I}\right\| / \Delta x\left(\mathrm{~s}^{-1}\right)$ & 25.8 & 0.0080 & 0.59 & 0.128 \\
\hline$\left\|\mathbf{u}^{I}\right\| \Delta x\left(\mathrm{~m}^{2} / \mathrm{s}\right)$ & 89690. & 4.2 & 8904. & 7520. \\
$\left\|\mathbf{u}^{I I}\right\| \Delta x\left(\mathrm{~m}^{2} / \mathrm{s}\right)$ & 129258. & 11.8 & 14222. & 13501. \\
\hline
\end{tabular}

Table 1: Grid and wind field statistics on full domain (top) and statistics on the transport zone defined on figure 1 (bottom).
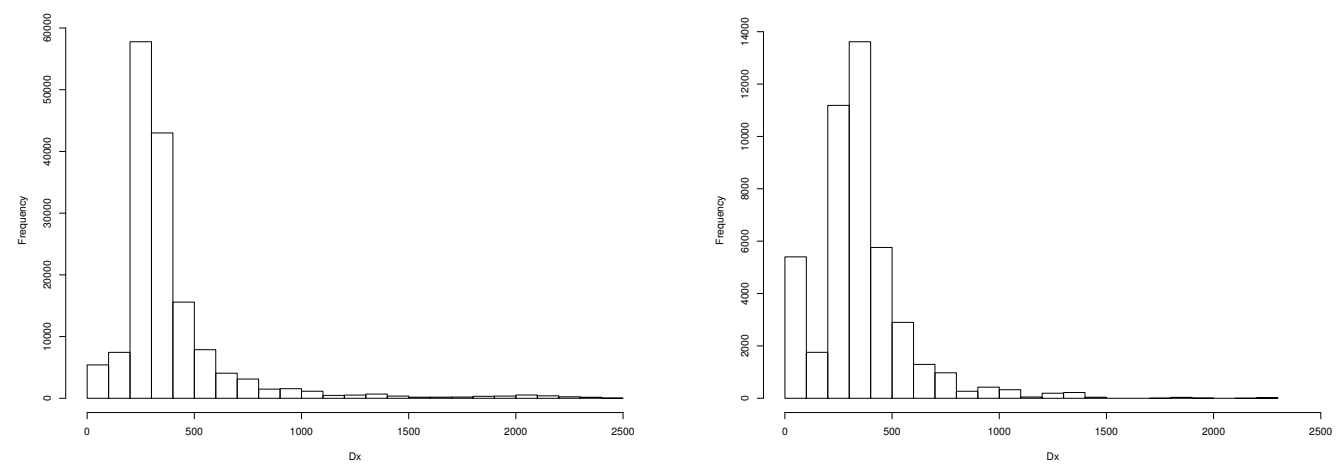

Figure 4: $\Delta x$ histogram on full domain (left) and on emission and transport zone (right).

indicators in both full domain and transport zone defined in figure 1. The computed indicators are: 1) thetahedra size (mean length of the six sides), $\Delta x ; 2$ ) velocity vector norm, $\|\mathbf{u}\| ; 3$ ) fraction $\|\mathbf{u}\| / \Delta x$; and 4) product $\|\mathbf{u}\| \Delta x$. Last two are closely related to dimensionless Courant, $C=\Delta t\|\mathbf{u}\| / \Delta x$, and Peclet, $P e=\|\mathbf{u}\| \Delta x /(2\|\mathbf{K}\|)$, cell numbers, which determine the behavior of numerical solvers.

From table 1 follows that differences between indicators computed in full domain 

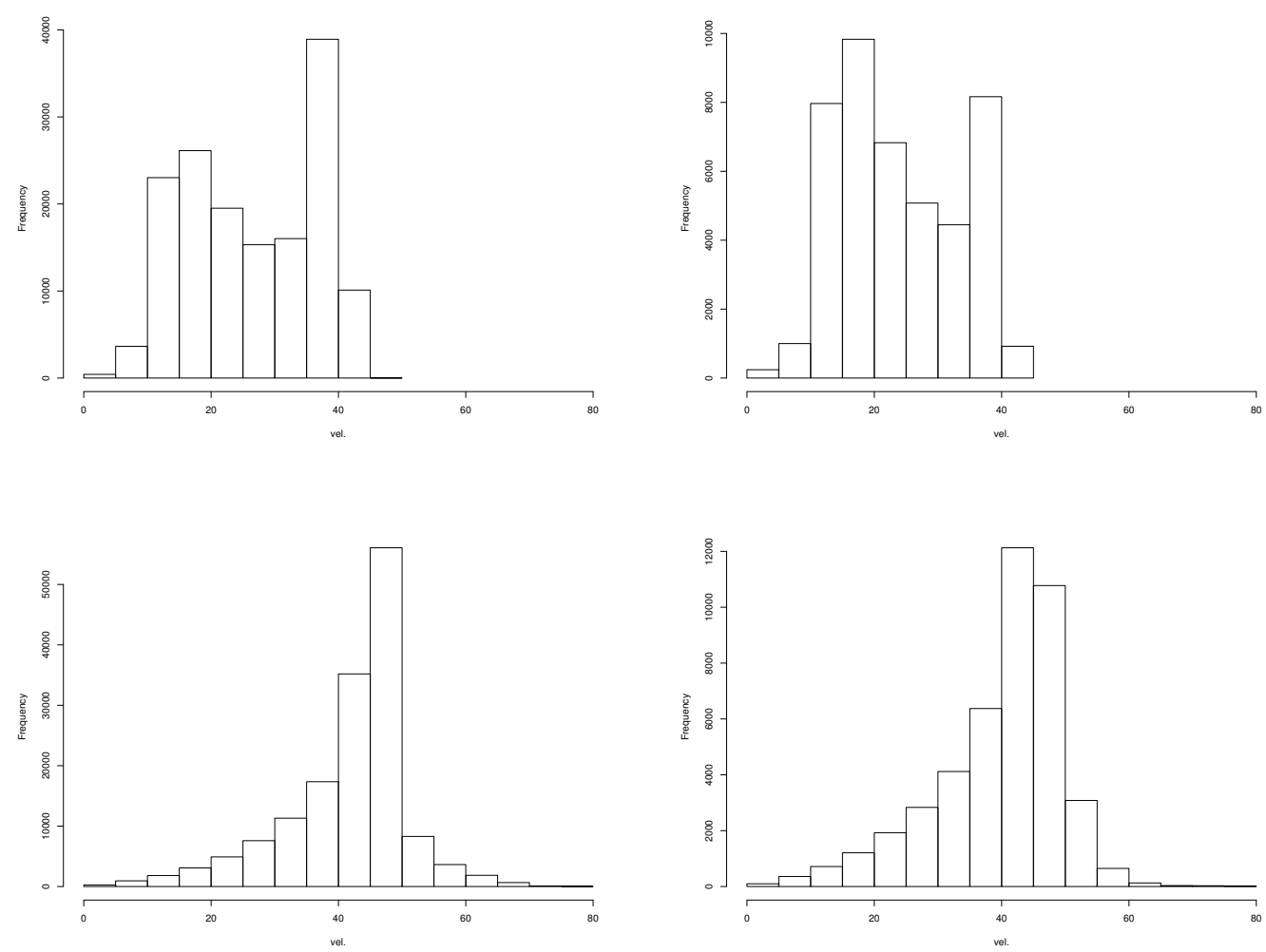

Figure 5: $\left\|\mathbf{u}^{I}\right\|$ (top) and $\left\|\mathbf{u}^{I I}\right\|$ (bottom) histograms on full domain (left) and on transport zone (right).

\begin{tabular}{|c|c|c|}
\hline$\Delta t_{r e f, 1,2,3}^{I}(\mathrm{~s})$ & \% elements with $C<1$ & Maximum $C$ \\
\hline 0.09 & $100 \%$ & 1 \\
0.9 & $99 \%$ & 10 \\
9 & $76 \%$ & 100 \\
\hline
\end{tabular}

\begin{tabular}{|c|c|c|}
\hline$\Delta t_{r e f, 1,2,3}^{I I}(\mathrm{~s})$ & \% elements with $C<1$ & Maximum $C$ \\
\hline 0.04 & $100 \%$ & 1 \\
0.4 & $99 \%$ & 10 \\
4 & $95 \%$ & 100 \\
\hline
\end{tabular}

Table 2: $\Delta t_{\text {ref }}$ values used in the transport - reaction simulations, case I (top) and case II (bottom). Maximum Courant numbers and accumulated percentage of elements with Courant number less than one are also shown.

and transport zone are small, except on maximum $\Delta x$ and $\left\|\mathbf{u}^{I I}\right\|$ which are significantly reduced.

Figure 4 shows $\Delta x$ histograms. Spatial discretization contain major number of 

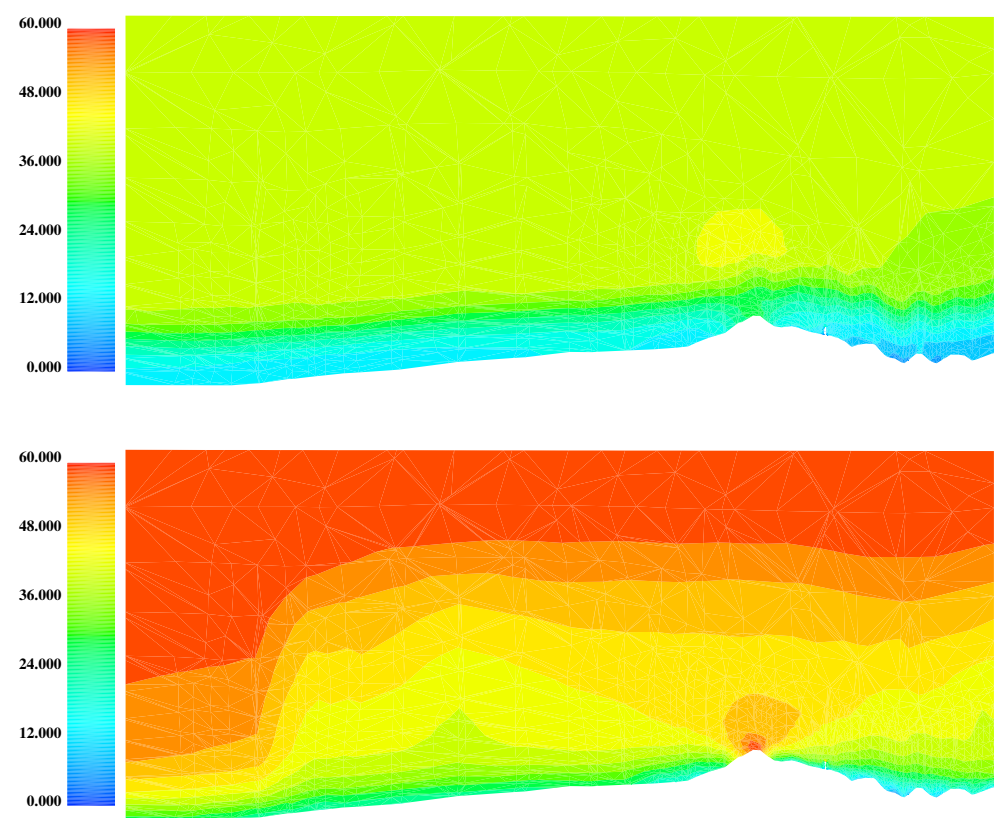

Figure 6: $\left\|\mathbf{u}^{I}\right\|$ (top) and $\left\|\mathbf{u}^{I I}\right\|$ (bottom) distribution slices.
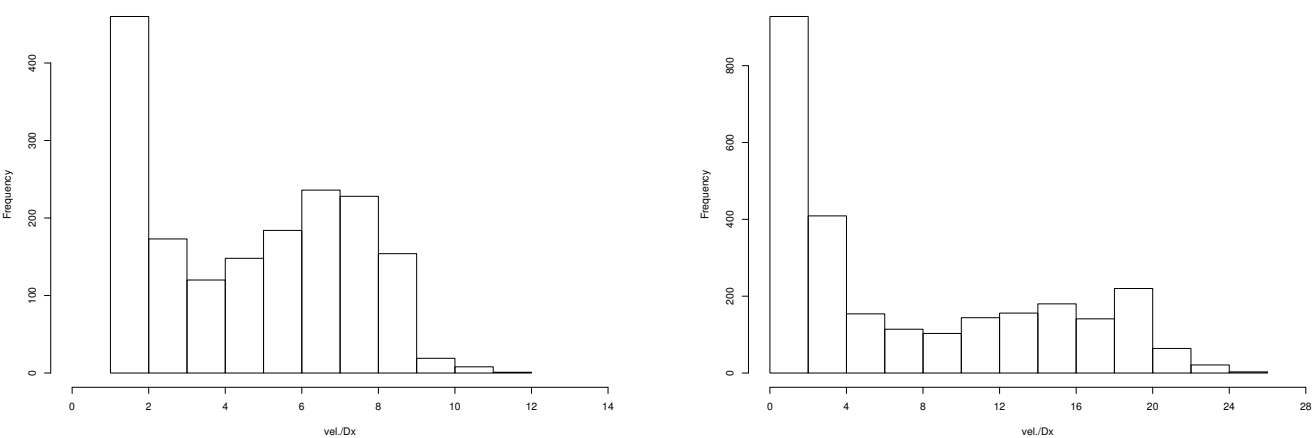

Figure 7: $\left\|\mathbf{u}^{I}\right\| / \Delta x$ (left) and $\left\|\mathbf{u}^{I I}\right\| / \Delta x$ (right) histograms on full domain. Only values greater than one are considered in both cases.

element sizes ranging from 200 to 400 meters, concentrating elements of less than 100 meters near source. However, figure 3 shows that in transport zone there are elements with horizontal sides about two kilometers, although its mean size is about hundreds of meters due their reduced height. These elements covers a flat terrain area, where the remeshing criteria used based on terrain curvature, does not activate enough.

Figure 5 shows velocity histograms. Wind velocity distribution of case I present two peaks in full domain, one in 20 and the other on $40 \mathrm{~m} / \mathrm{s}$, but on transport zone the 

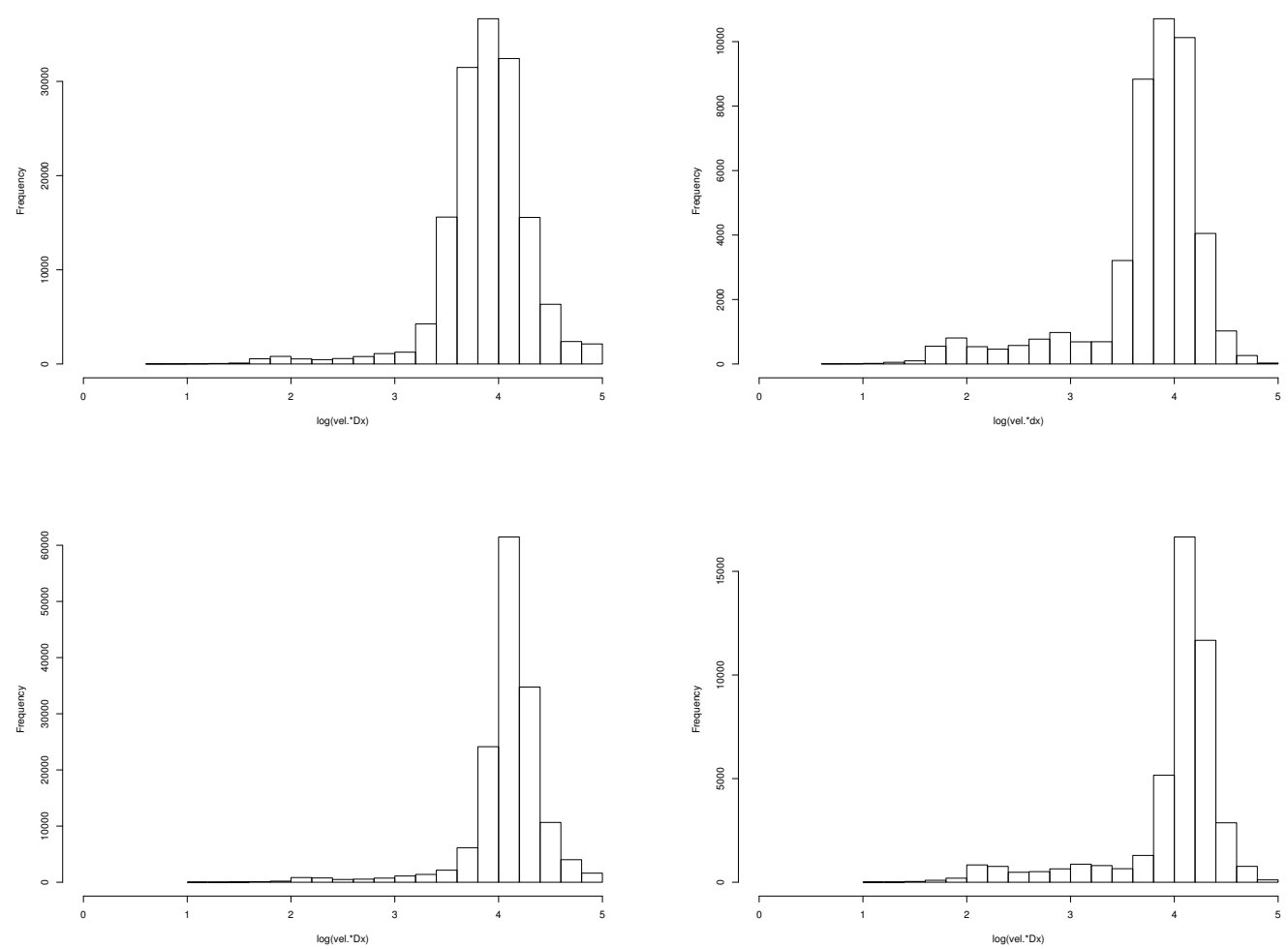

Figure 8: $\log \left(\left\|\mathbf{u}^{I}\right\| \Delta x\right)$ (top) and $\log \left(\left\|\mathbf{u}^{I I}\right\| \Delta x\right)$ (bottom) histograms on full domain (left) and on emission and transport zone (right).

important one is $20 \mathrm{~m} / \mathrm{s}$. Figure 6 show that high velocities are on high altitudes, thus they do not affect the transport. Wind velocity distribution of case II has just a peak value between 40 and $50 \mathrm{~m} / \mathrm{s}$ in both full domain and transport zone. Figure 6 show that in case II high velocities concentrate on both ridges and high altitudes. Recall that velocities of case II correspond to an exceptional situation of 150-180 Km/h, with 216 $\mathrm{Km} / \mathrm{h}$ peaks, on upper parts of the mountains.

Figure 7 shows $\|\mathbf{u}\| / \Delta x$ histograms, for values greater than one. Table 1 shows that mean an median are much lower than one, thus just a small part of the domain is represent in figure 7 . Both cases share the distribution tail, situation directly related with the use of the same mesh. The upper part of the tail correspond to the elements with lower $\Delta x$, the top part of the stack. These histograms let adjust time step to the requierements of time integration scheme. Crank Nicolson scheme has no restrictions on time step for stability, but values of Courant not much higher that one are recommended for accuracy. Table 2 shows three reference $\Delta t$ for each case. The are found imposing Courant number equal to 1,10 and 100 to the most demanding element. The percentage of elements with $C<1$ are also indicated.

Last mesh and wind field analysis is related with the Peclet number. Figure 8 shows 

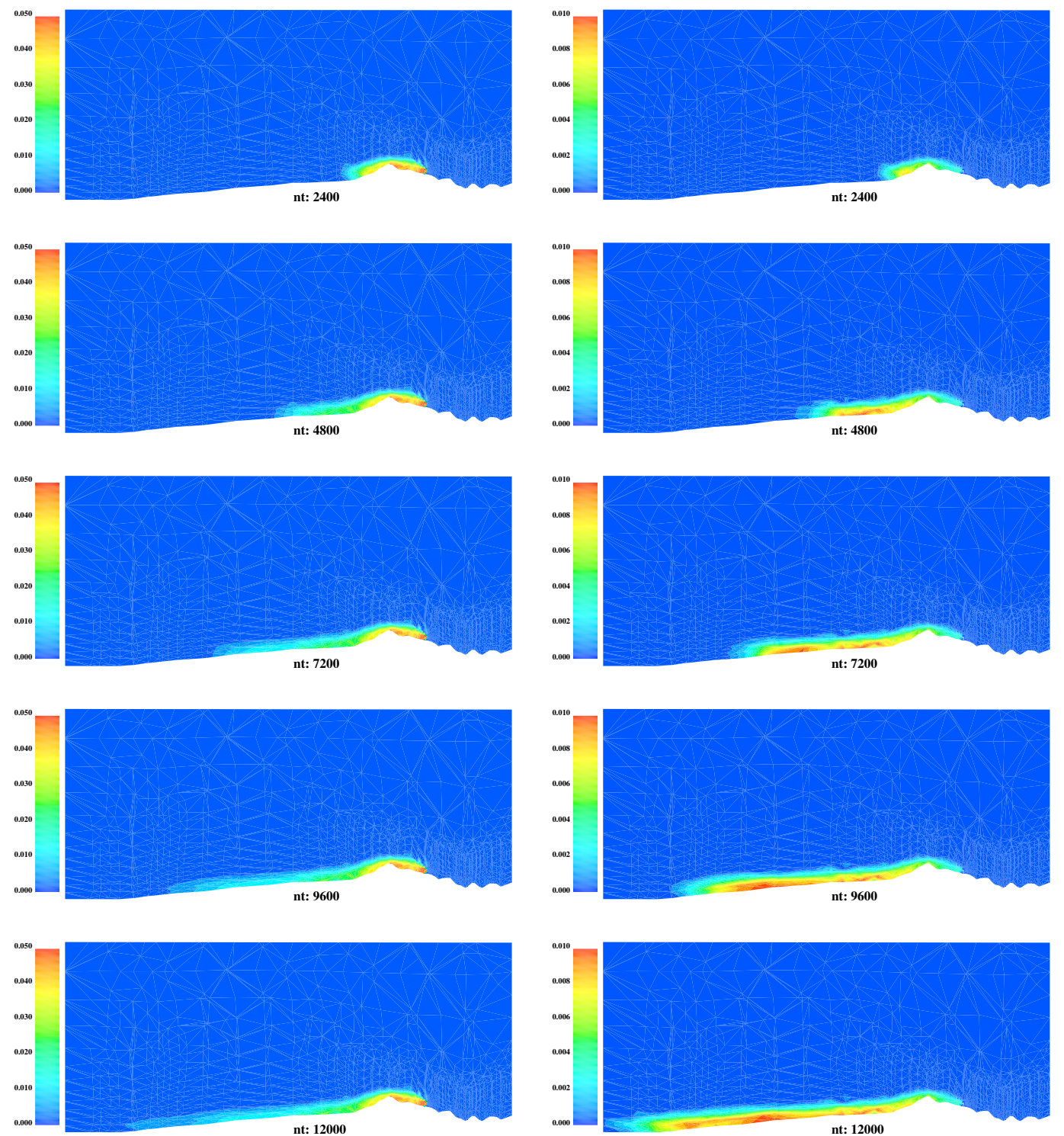

Figure 9: Case I. Primary (left) and secondary (right) pollutant evolution, with $\Delta t_{r e f, 1}^{I}$.

$\log (\|\mathbf{u}\| \Delta x)$ histograms. Major part of elements have this indicator in the range $10^{3}-$ $10^{4}$, which correspond to Peclet numbers $10-10^{2}$, in the case I, and in the range $10^{4}$ $-10^{5}$, thus with Peclet numbers ranging $10^{2}-10^{3}$, in the case II, although differences between both cases are reduced. These magnitudes indicate that the problem is highly convective, especially the case II. Remarkably both wind fields has lower numbers near the emission stack, thus less convective behavior can be expected there. 

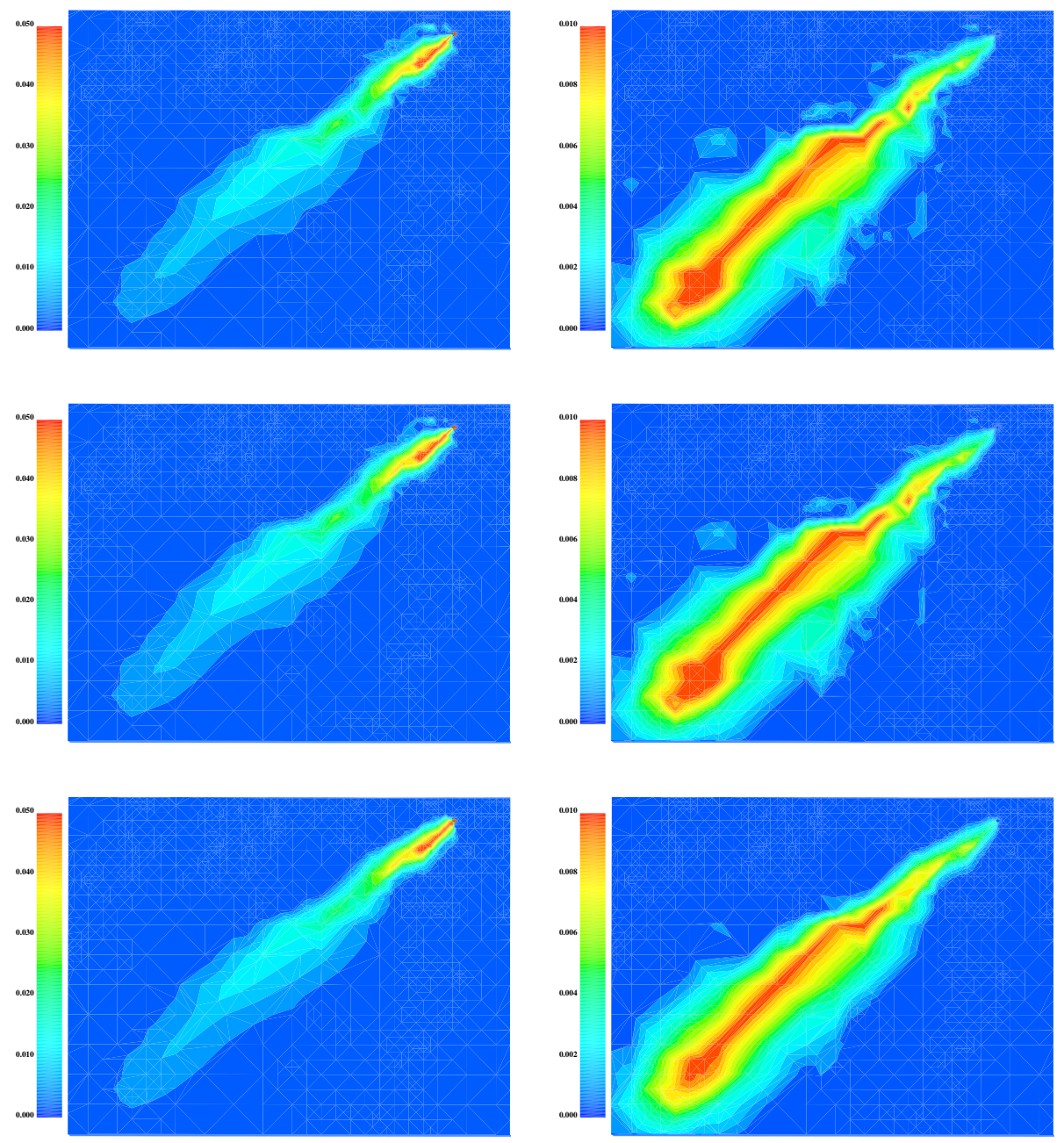

Figure 10: Case I. Primary (left) and secondary (right) ground views with $\Delta t_{r e f, 1}^{I}$ (top), $\Delta t_{r e f, 2}^{I}$ (medium) and $\Delta t_{r e f, 3}^{I}$ (bottom).

\section{Transport and reaction simulations}

In this section, numerical solution of transport and reaction reference problems (case I and II) is presented. Specific attention is given to the influence of time step in the results. Each case is solved until a final time corresponding to $\Delta t_{r e f, 1}$ multiplied by 12000. Note that as $\Delta t_{r e f, 1}^{I}$ is approximately double than $\Delta t_{r e f, 1}^{I I}$ and velocity of case I is half of that of case II, the maximum distance transported will be similar in both cases.

Figure 9 summarizes the evolution of primary and secondary pollutants for case I computed with $\Delta t_{r e f, 1}^{I}$. It shows how primary pollutant moves because of convection and its concentration decrease because reaction and diffusion; and how secondary pollutant increases concentration due to reaction, and moves because of convection 

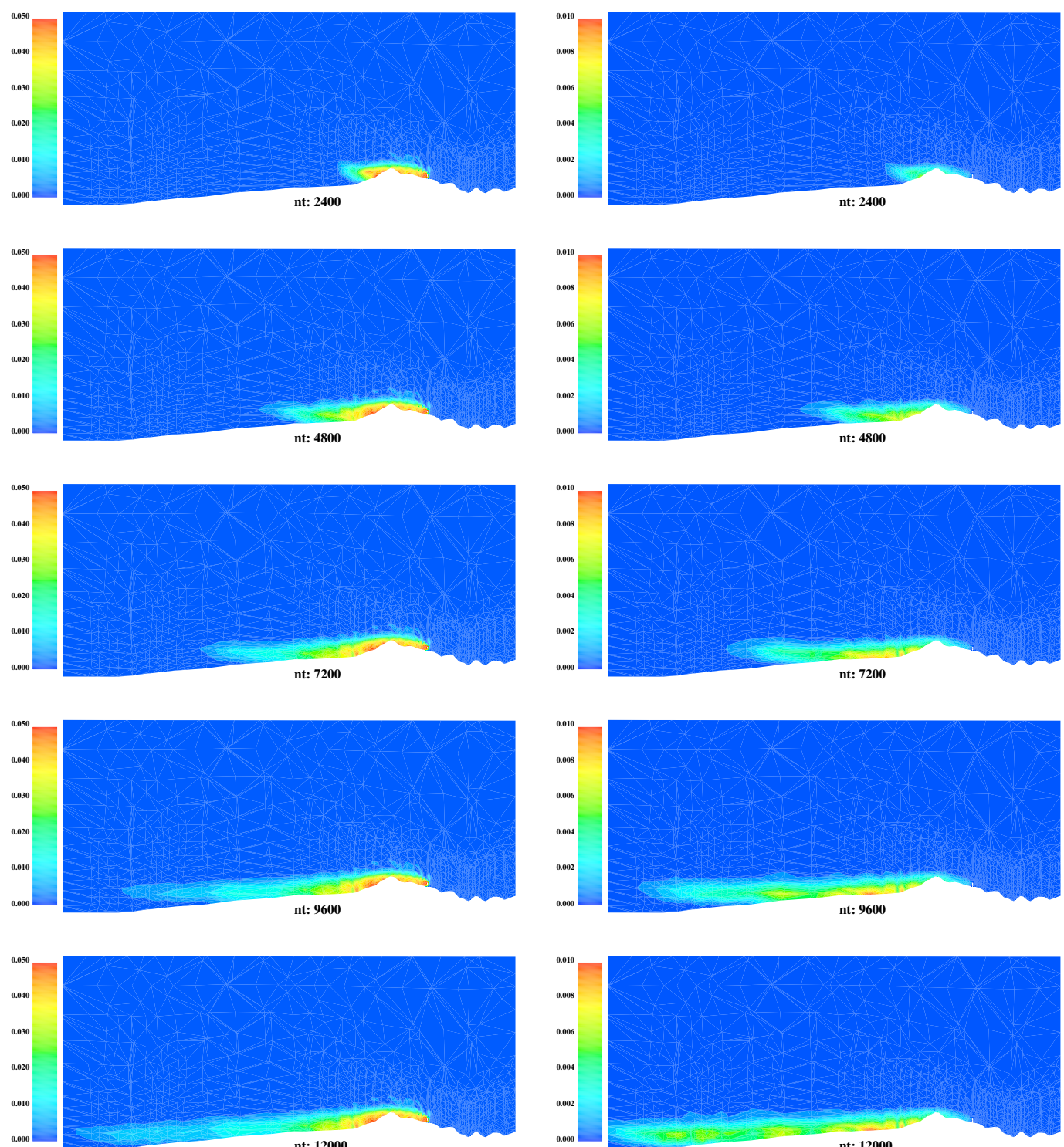

Figure 11: Case II. Primary (left) and secondary (right) pollutant evolution, with $\Delta t_{\text {ref }, 1}^{I}$.

and diffusion. Pollutant moves close to ground due to wind field distribution.

One of the key outputs of transport - reaction analysis are the ground level concentrations. Figure 10 shows ground level concentration of primary and secondary pollutants computed with $\Delta t_{r e f, 1}^{I}, \Delta t_{r e f, 2}^{I}$ and $\Delta t_{r e f, 3}^{I}$. Results with first and second time step are very similar, presenting some small spurious oscillations on plume sides. Instead, results with larger time steps, as the third one used as example, are too smoothed.

Figures 11 and 12 show the same analysis for the case II wind field. As in case I 

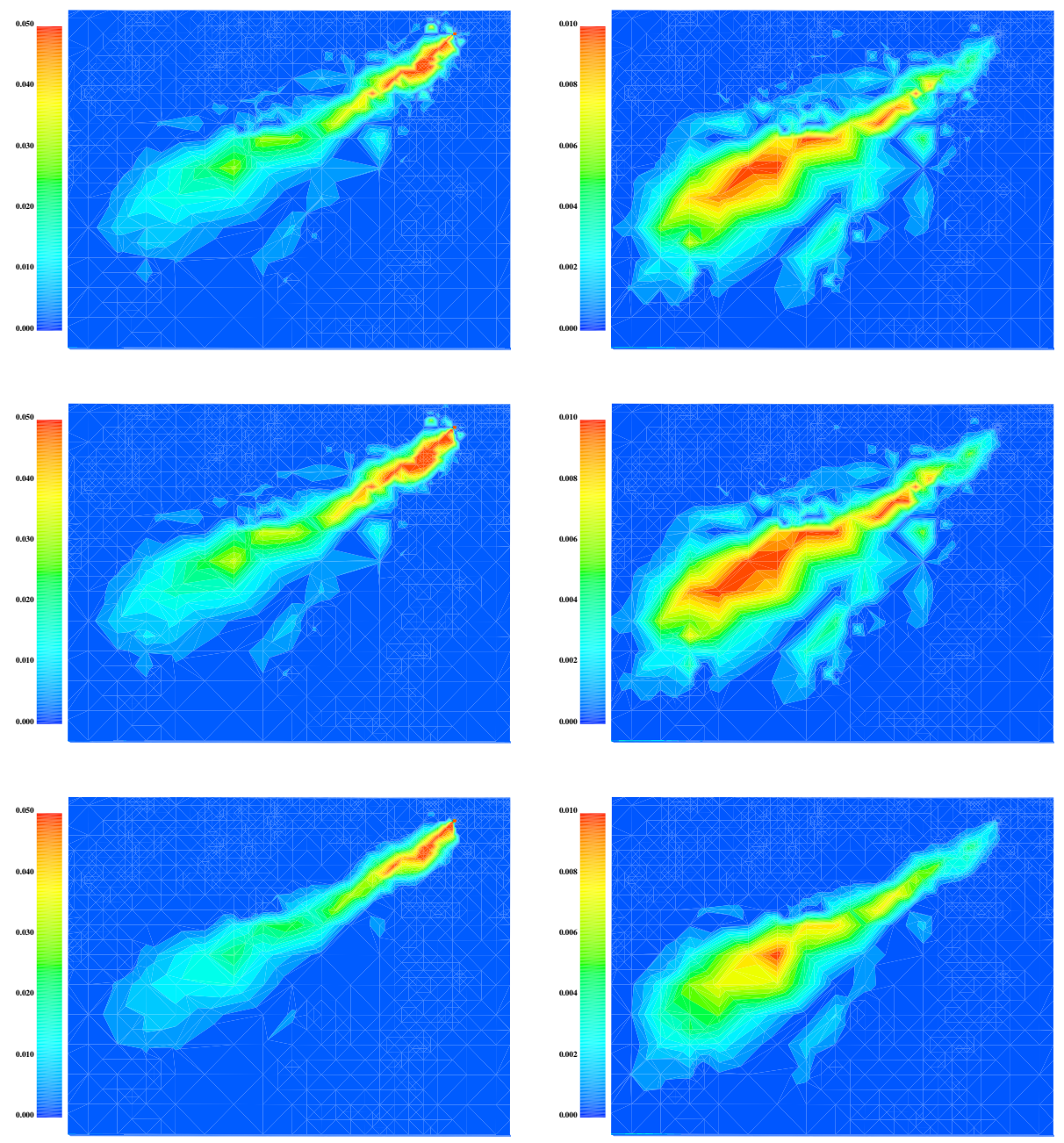

Figure 12: Case II. Primary (left) and secondary (right) pollutant ground views with $\Delta t_{r e f, 1}^{I I}$ (top), $\Delta t_{r e f, 2}^{I I}$ (medium) and $\Delta t_{r e f, 3}^{I I}$ (bottom).

example, results with maximum Courant numbers equal to 1 and 10 are equivalent, but with larger time steps the solution is too smoothed. The main difference with respect to case I is the presence of larger spurious oscillations, significant both in vertical slide and in ground level.

Oscillations start close to emission stack, but they are mainly in the zone with large horizontal size elements. Provided that the case I simulation present less spurious oscillations and lower Peclet numbers that case II (compare Peclet number distributions in figure 8 ) one possible way to improve case II results would be to remesh in transport zone. Remarkably, the simple approach presented here gives correct results except for extremely windy situations.

Last analysis presented involves RIVAD nonlinear model. Figure 13 shows the solution of primary and secondary pollution for case I wind conditions at final time 

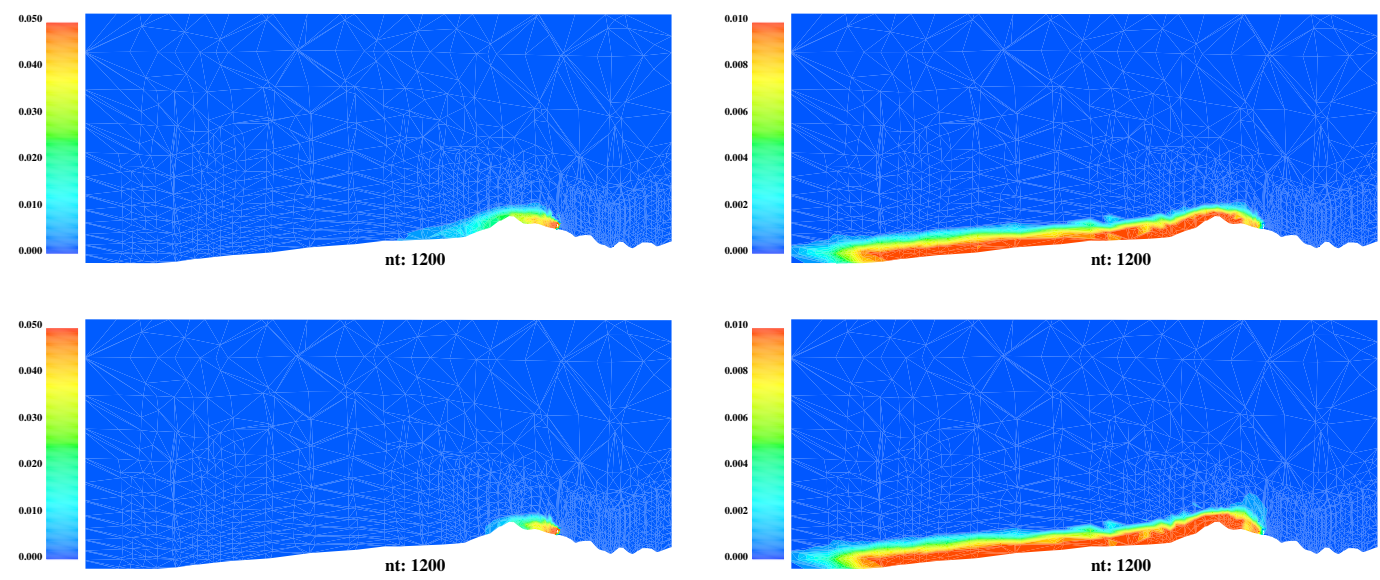

Figure 13: RIVAD model. Case I. Primary (left) and secondary (right) pollutant evolution, with $\Delta t_{r e f, 2}^{I}$. Two models are considered: $\alpha_{R \max }=5 \alpha$ (top) and $50 \alpha$ (bottom).

simulation, 1200 time steps of $\Delta t_{r e f, 2}^{I}$ size. Model parameters $\delta_{1,3}=1, \alpha_{1,3}=\alpha$ and $\alpha_{R \max }=5 \alpha$ and $50 \alpha$ are considered. Note that as with these model parameters both primary and both secondary pollutants will present the same behavior, for this reason only one primary and one secondary pollutant evolutions are shown in figure 13.

The influence of nonlinearity becomes clear when comparing figure 13 with bottom graphics of figure 9. Higher reaction rates for low concentration of primary pollutants of nonlinear model produces reduced primary plumes and larger secondary ones. Size of primary plumes is directly related with $\alpha_{R \max }$. Larger values of $\alpha_{R \max }$ imply faster chemical transformation at low values of concentration, and therefore smaller primary pollutant plumes.

\section{Conclusions}

A review of last tendencies in air pollutant simulation has shown that the Finite Element Method could represent a significant improvement to puff models for simulations involving punctual emissions sources, specially when used embedded into Eulerian grid models. Tridimensional discretizations including details of punctual sources present, at least, element sizes ranging three orders of magnitude: From few meters near the source to few kilometres far away. Therefore special attention is needed to properly distretize the spatial domain and time integrate the material model. The work presented here focuses in these topics.

Linear and nonlinear chemistry is considered. Test examples involve highly complex topography and two wind fields, corresponding to strong and extremely strong situations. Wind field is computed first, using a mass consistent model. After that, linear transport - reaction problems are solved with a Least Square approach and Crank-Nicolson time integration. Nonlinear problems are solved with a chemical - 
transport splitting scheme and a Rosembrock time integrator for the chemical step. The same mesh, adapted to terrain curvature, is used in both wind and transport reaction problems.

Expected results have been found with both with linear and nonlinear models. Detailed discretization of punctual sources have not implied a strong requirement on time integration stepping, confirming the possibilities of the proposed approach. Refinement of meshes in plane terrain zones will improve accuracy and avoid some spurious oscillations found in extremely windy situations.

\section{References}

[1] A.A. Jennings and S.J. Kuhlman. An air pollution transport teaching module based on GAUSSIAN MODELS 1.1. Environmental Modelling and Software, 12(2-3):151-160, 1997.

[2] J.F. Hernández, L. Cremades, and J.M. Baldasano. Dispersion modelling of a tall stack plume in the Spanish Mediterranean coast by a particle model. Atmospheric Environment, 29(11):1331-1341, 1995.

[3] C.P.A. Bourque and P.A. Arp. Simulating sulfur dioxide plume dispersion and subsequent deposition downwind from a stationary point source: A model. Environmental Pollution, 91(3):363-380, 1996.

[4] M.J. Souto, J.A. Souto, V. Pérez-Muuzuri, J.J. Casares, and J.L. Bermúdez. A comparison of operational Lagrangian particle and adaptive puff models for plume dispersion forecasting. Atmospheric Environment, 35:2349-2360, 2001.

[5] J.S. Scire, D.G. Strimaitis, and R.J. Yamartino. A User's Guide for the Calpuff dispersion Model (version 5). Earth Tech., Inc, Concord, MA., 2000.

[6] Z. Zlatev. Computer treatment of large air pollution models. Kluver Academic Publishers, Dordrecht., 1995.

[7] R.E. Morris, G. Yarwood, C.A. Emery, and G.M. Wilson. Recent advances in photochemical air quality modeling using the CAMx Model: Current update and ozone modeling of point source impacts. In AWMA, 2002.

[8] M. Taghavi, S. Cautenet, and J. Arteta. Impact of a highly detailed emission inventory on modeling accuracy. Atmospheric Research, 74:65-88, 2005.

[9] R.S. José, J.L. Pérez, and R.M. González. An operational real-time air quality modelling system for industrial plants. Environmental Modelling and Software (in press), 2006.

[10] CAMx Comprehensive Air quality Model with extensions. User's Guide v. 4.20. ENVIRON International Corporation. Novato, California., 1999.

[11] User's Guide to the variable-grid Urban Airshed Model (UAM-V). Systems Applications International, Inc., ICF Consulting, Inc., San Rafael, California., 1999.

[12] D.W. Byun and J.K.S. Ching. Science algorithms of the EPA models-3 Community Multiscale Air Quality (CMAQ) modelling system. EPA/600/R-90/030. 
Atmospheric Modeling Division. U.S. Environmental Protection Agency. Washington DC., 1999.

[13] G.M. Hidy. Multiscale impact of fuel consumption on air quality. Energy and Fuels, 16:270-281, 2002.

[14] T.B. Ryerson and M. Trainer. Observations of ozone formation in power plant plumes and implications for ozone control strategies. Science, 292:719-723, 2001.

[15] S. Lee, H.J.S. Fernando, M. Princevac, D. Zajic, M. Sinesi, J.L. MacCulley, and J. Anderson. Transport and Diffusion of Ozone in the Nocturnal and Morning Planetary Boundary Layer of the Phoenix Valley. Environmental Fluid Mechanics, 3:331-362, 2003.

[16] D.L. Mauzeralla, B. Sultan, N. Kim, and D.F. Bradford. NOx emissions from large point sources: variability in ozone production, resulting health damages and economic costs. Atmospheric Environment, 39:2851-2866, 2005.

[17] I. Lagzi, D. Kármán, T. Turányi, A.S. Tomlin, and L. Haszpra. Simulation of the dispersion of nuclear contamination using an adaptive Eulerian grid model. Journal of Environmental Radioactivity, 75:59-82, 2004.

[18] N.N. Ahmad, D.P. Bacon, M.S. Hall, and A. Sarma. Application of the multidimensional positive definite advection transport algorithm (MPDATA) to environmental modelling on adaptive unstructured grids. Int. J. Numer. Meth. Fluids (in press), 2006.

[19] A.S. Tomlin, S. Ghorai, G. Hart, and M. Berzins. 3-D Multi-scale air pollution modelling using adaptive unstructured meshes. Environmental Modelling and Software, 15:681-692, 2000.

[20] G. Montero, R. Montenegro, and J.M. Escobar. A 3-D diagnostic model for wind field adjustment. Journal of Wind Engineering and Industrial Aerodynamics, 74 76:249-261, 1998.

[21] R. Montenegro, G. Montero, J.M. Escobar, E. Rodríguez, and J.M. GonzálezYuste. 3-D Adaptive Wind Field Simulation Including Effects of Chimney Emissions. In WCCM VI in conjunction with APCOM'04, Beijing, China. Tsinghua University Press and Springer-Verlag, 2004.

[22] G. Montero, E. Rodríguez, R. Montenegro, J.M. Escobar, and J.M. GonzálezYuste. Genetic algorithms for an improved parameter estimation with local refinement of tetrahedral meshes in a wind model. Advances in Engineering Software, 36:3-10, 2005.

[23] L.L. Burrell, L.Q. Tang, and T.T.H. Tsang. On a Least-Squares Finite Element method for advective transport in air pollution modeling. Atmospheric Environment, 29:1425-1439, 1995.

[24] J.L. Wright, B. Chowdhury, L.Q. Tang, and T.T.H. Tsang. Grid refinement tests of a least-squares finite element method for advective transport of reactive species. Environmental Modelling and Software, 12:289-299, 1997.

[25] A. Huerta and J. Donea. Time-accurate solution of stabilized convectiondiffusion-reaction equations: I. Time and space discretization. Communications in Numerical Methods in Engineering, 18(8):565-573, 2002. 
[26] A. Huerta, B. Roig, and J. Donea. Time-accurate solution of stabilized convection-diffusion-reaction equations: II. Accuracy analysis and examples. Communications in Numerical Methods in Engineering, 18(8):575-584, 2002.

[27] A. Rodríguez-Ferran and M.L. Sandoval. Numerical performance of Incomplete Factorizations for 3D transient Convection-Diffusion problems. Advances in Engineering Software (in press), 2006.

[28] D.L. Ropp, J.N. Shadid, and C.C. Ober. Studies of the accuracy of time integration methods for reaction-diffusion problems. Journal of computational physics, 194:544-574, 2004.

[29] J.G. Verwer, E.J. Spee, J.G.Blom, and W.H. Hundsdorfer. A second Order Rosenbrock Method Applied to Photochemical Dispersion Problems. Centrum voor Wiskunde en Informatica, 1997.

[30] R. Montenegro, G. Montero, J.M. Escobar, E. Rodríguez, and J.M. GonzálezYuste. Tetrahedral mesh generation for environmental problems over complex terrains. Lecture Notes in Computer Science, 2329:335-344, 2002.

[31] J.M. Escobar, E. Rodríguez, R. Montenegro, G. Montero, and J.M. GonzálezYuste. Simultaneous untangling and smoothing of tetrahedral meshes. Comput. Methods Appl. Mech. Engrg., 192:2775-2787, 2003. 